\title{
LECTURA DE CONTEXTO: LA EDUCACIÓN POPULAR COMO PRÁCTICA LIBERTARIA ${ }^{2}$
}

THE READING OF CONTEXT:

POPULAR EDUCATION AS A LIBERTARIAN PRACTICE.

Recibido: mayo de 2012 - Revisado: octubre de 2012 - Aceptado: 30 de noviembre de 2012

Por: Diego Alejandro Muñoz Gaviria ${ }^{1}$.

\section{RESUMEN:}

El presente artículo de investigación pretende presentar la Educación Popular como una dinámica de la tradición pedagógica y movimiento cultural, resaltando su carácter diverso, multidireccional, no deductivo, es decir que, no existe un momento fundacional en el que se propusieron unos principios generales, unas bases conceptuales o doctrinales, desde las cuales se generaron unas prácticas pedagógicas y políticas posteriores y lo que ha existido es una suerte de campo educativo - popular donde existen diferentes perspectivas de lo popular.

\section{PALABRAS CLAVE:}

Educación popular, tradición político-pedagógica, pedagogía.

\section{ABSTRACT:}

This research article seeks to present the concept of popular education as a dynamic of pedagogical tradition and cultural movement, emphasizing its diverse, multidirectional, nondeductive character; that is to say, that there is not a foundational moment in which some general principles, conceptual or doctrinal foundations, were set out, from which subsequent political and pedagogical practices were generated and what there has been is a sort of popular educational field - where there are different perspectives of what is popular.

\section{KEY WORDS AND EXPRESSIONS:}

Popular education, political-pedagogical tradition, and pedagogy.

\footnotetext{
${ }^{1}$ Sociólogo, Especialista en Contextualización Psicosocial del Crimen, Magíster en Psicología de la Universidad de San Buenaventura Medellin. Estudios Doctorales en Ciencias Sociales: Niñez y Juventud de la Universidad de Manizales y el CINDE. Estudiante del doctorado en Filosofia de la UPB. Director del Grupo Interdisciplinario de Estudios Pedagógicos de la Universidad de San Buenaventura, y miembro del Grupo sobre Formación y Antropología Pedagógica Formaph de la Universidad de Antioquia. E-mail: diegomudantehotmail.com
} 


\section{Introducción.}

"La pedagogía del oprimido que, en el fondo, es la pedagogía de los hombres que se empeñan en la lucha por su liberación, tiene sus raíces en la transformación. Y debe tener, en los propios oprimidos que se saben o empiezan a conocerse críticamente como oprimidos, uno de sus sujetos".

(Freire, 1970,p.46).

En la comprensión de las realidades contextuales de la educación popular (EP) es importante asumir algunas consideraciones históricas, epistemológicas, politicas y pedagógicas que pueden girar en torno al siguiente supuesto: La EP es una tradición político pedagógica de corte crítico configurada histórica y contextualmente en América Latina. De allí que tenga una identidad propia, marcada por una realidad histórica y socio-política específicamente Latinoamericana (FUENTES,2008).. Por lo anterior, la EP asume realidades contextuales según matices como: procesos de lucha contra las dictaduras y a los proceso de democratización en el Cono Sur; el acento en lo étnico y lo indígena en los países andinos; o las iniciativas de organizaciones independientes frente a los partidos políticos en las actuales democracias neoliberales. Así, la dinámica de la EP como tradición pedagógica y como movimiento cultural no es unidireccional ni deductiva, es decir, no existe un momento fundacional en el que se propusieron unos principios generales, unas bases conceptuales o doctrinales, desde las cuales se generaron unas prácticas pedagógicas y políticas posteriores. Lo que ha existido es una suerte de campo educativo - popular (Mejía, Awad, 2004) donde existen diferentes perspectivas de lo popular.

Con el interés de fundamentar estas ideas, el presente escrito pretende tematizar los siguientes items:

\section{Contexto de emergencia.}

Podríamos decir que el momento fundacional de la EP se presenta a finales de los 70, década en la cual al EP consigue ubicarse como una tradición pedagógica soportada en la obra del pedagogo Paulo Freire (brasileño), con expresiones pedagógicas como: educación liberadora, educación para la libertad, educación concientizadora, educación dialogal, entre otras., sin embargo, su reflexión educativa y su propuesta pedagógica influyó notablemente a la EP, principalmente en la fundamentación teórica y práctica del diálogo existente entre pedagogía y politica (Torres, 2011).

Como antesala a esta consolidación histórica de la EP, se tiene las discusiones y prácticas que se plantea la izquierda latinoamericana a partir de la segunda mitad del siglo XX, en torno a temas como la dependencia, el imperialismo y la identidad de los pueblos. Como hecho significativo se cuenta con el triunfo de la Revolución cubana en 1959, que contribuye a repensar y desarrollar un socialismo latinoamericano, capaz de aprovechar lo mejor del marxismo científico europeo, pero que a la vez cuestiona los dogmas que escapan a nuestra realidad y propenden por un socialismo raizal; el surgimiento de la Teología de la Liberación como postura teológica latinoamericana, que coloca a la izquierda en el desafío de la construcción política a partir de los sectores populares y sus necesidades; la crisis del modelo soviético, que viene a plantear la importancia de articular el socialismo con formas democráticas de organización y lucha, además de la necesaria conciencia histórica de los pueblos; Nicaragua y la revolución Sandinistas, representará el momento cumbre donde lo 
popular, lo revolucionario, lo socialista, lo cristiano se van a encontrar. Es en ese contexto, donde se empieza a hablar de EP más claramente (Mendoza y Nuñez,2002).

En este contexto histórico se fue configurando la EP, con aportes de propuestas sociales como la Investigación Acción participación, la Comunicación Popular, la teoría de la dependencia, los estudios de modernidad - colonialidad, entre otros. Todas estas propuestas históricas, culturales, pedagógicas y politicas van a unirse en un enfoque común a través de las prácticas, espacios y actores ubicados en el campo de la EP.

\section{Metodologia.}

El presente artículo de investigación se enmarca dentro del proyecto "de Tijuana a la Patagonia" cuanta con un enfoque cualitativo que desde el paradigma crítico social exige un contacto directo con la realidad, una confrontación teoría - praxis, centra la construcción de teorías de forma inductiva a partir de un conjunto de datos, dicho proceso tiene como finalidad esencial abordar e interpretar la realidad para transformarla, puesto que ésta depende de las circunstancias, es una construcción y reconstrucción intersubjetiva, donde la acción comunicativa es la base del conocimiento.

Para la recolección de información, se utilizaron tres instrumentos denominados Matriz de categorías que se construyó a partir del fundamento teórico y de discusiones grupales para aplicare en el rastreo y análisis bibliográfico y el segundo instrumento, consiste en la discusiones en grupos de interés indagando sobre los aspectos relacionados con la educación popular a partir de la experiencia personal, el proceso de construcción académica y desempeño profesional de cada participante.

Resultados - discusiones.

¿Qué es la educación popular?.

La historia de la Educación Popular no es lineal, no se comprende desde la sucesión del cambio de una propuesta por otra. Por ello, las propuestas pedagógicas de la EP se encuentran mezcladas las unas con las otras. Podría decirse que el campo de la EP se caracteriza por un tacto pedagógico diferente a aquel producido por las élites y su educación bancaria, reproductor del sistema social de injusticia, que genera seres humanos que se acomodan a la sociedad sin transformarla, sin ser sujetos históricos de cambio

La EP, es una estrategia de política cultural, desde la cual agenciar acciones pedagógicas y politicas que colaboran en la construcción de una alternativa cultural y politica de sociedad justa y equitativa, donde se puedan pensar otros mundos posibles, en los cuales, quepan otros mundos (Lorenzo, 2008). Una pedagogía del oprimido que reivindica el empoderamiento de sujetos y pueblos, y que deja en la distancia las viejas consignas intervencionistas apoyadas en la idea de propuestas pedagógicas "para" los excluidos, dejando por fuera la opresión y sus causas como el objeto de reflexión de los oprimidos (Carballo, 2008)

Pensar la EP como una pedagogía del oprimido, nos permite ubicar al menos tres acercamientos conceptuales a ésta: una definición en términos del empoderamiento de los pueblos (política), otra que pone el acento sobre la formación - autoeducación de los sectores populares (pedagógica), y finalmente una definición que insiste sobre la reconstrucción del tejido social (sociológica). 
- La EP en clave política: La EP es un proceso social mediante el cual los sectores populares llegan a convertirse en el sujeto histórico - mesiánico, gestor y protagonista de un proyecto liberador que encarne sus propios intereses de clase. Por ello, la EP es vista como parte y apoyo a un proceso político mediante el cual los sectores populares, a partir de su práctica social, van construyendo y consolidando su propia hegemonía politica, es decir, desarrollando las condiciones subjetivas- la conciencia política y la organización popular- que les hará posibles la construcción de su propio proyecto histórico.

Esta idea de la política no se desvincula de una perspectiva ética, la EP es ético - política, dada su profunda convicción humanista, en la cual ninguna emancipación política podría darse, sin una emancipación humana.

- La EP en clave pedagógica: La EP es un campo conceptual y práctico donde los sectores populares construyen colectivamente - dialógicamente su saber, sus formas de aprender y comprender los hechos de la vida social. Es el saber que emerge de la experiencia de vida y de lucha de los movimientos sociales y que es elaborado por ellos mismos, que legitima su poder de transformar la sociedad: es ese saber que aumenta la capacidad de discernir, rechazar, resistir y alterar las reglas de dominación, y que fortalece su poder de decidir cuáles son las luchas y formas de organización más capaces de concretar nuevas reglas de vida social. Por ello, la formación en este campo de la EP, sólo puede comprenderse como emancipación y la educación como transformación social.

La lectura pedagógica de la EP, desmiente las ideas educativas - formativas domesticadoras de las tradiciones pedagógicas eurocentradas, a saber: las ciencias de la educación francófonas, la ciencia de la educación alemana y las teorías curriculares anglosajonas. El común denominador de estas tradiciones es la confianza en el dispositivo escuela y su funcionalidad en las modernas sociedades industriales. Para la EP, el principal aporte pedagógico latinoamericano es la crítica a la escuela como máquina de educar, y la reivindicación de los círculos de cultura y la educación como práctica de la libertad, como centro de sus propuestas pedagógicas. Así, lo pedagógico en la EP no es sinónimo de un saber para la reproducción social, sino, un saber de los oprimidos para su concienciación y liberación.

- La EP en clave sociológica: La EP es entendida como educación para los movimientos sociales, tiene por objeto la recreación de las bases de sociabilidad, de solidaridad en una configuración social específica. La educación para los movimientos sociales es una praxis pedagógica programada e informal, orientada a complementar el conjunto de actividades educativas y formativas existentes en la sociedad.

En clave sociológica, la EP comparte con la sociología de la liberación latinoamericana la defensa de la solidaridad como pegante moral de lo social (Fals - Borda, 1970) A diferencia y en contraposición con las posturas neoliberales, que asumen como reducción antropológica al ser humano como ser egoísta, la EP postula y defiende la solidaridad como reivindicación antropológica de un ser humano altérico o en relación con los otros.

Para resumir, se podría pensar en la EP como un proceso de formación y educación desde la reivindicación de los sectores populares para que con ellos, a través de una acción transformadora, se logre romper con los esquemas de dominación (opresores y oprimidos) 
con el objetivo de configurar una Sociedad de sujetos emancipados y solidarios. Pero aún hace falta esclarecer qué es lo educativo desde esta mirada pedagógica.

¿Qué es lo educativo?.

En las tradiciones pedagógicas críticas, entre ellas la EP, la pregunta por la educación es la pregunta por temas como la reproducción, la resistencia y la liberación. En la primera acepción, la educación parece quedar rezagada a una mirada crítica que sólo ve en ella la reproducción de intereses de clase y llamados económicos de producción; para esta postura la educación es la expresión de la superestructura como parte de la ideología y como aparato ideológico del Estado. Una segunda posición, cercana al tema de la resistencia y la liberación, postula la incidencia de la educación en la transformación social; es un espacio donde se juegan grandes tensiones sociales y políticas. Se utiliza la palabra educación como sinónimo de "proceso político pedagógico". Para la mira pedagógica de la EP a la educación, existen múltiples interacciones entre las diferentes esferas de la práctica social de modo que el proceso educativo no está completamente determinado por lo económico, ni tampoco autónomo. Por ello, es conveniente en el horizonte de la pregunta por la educación popular tener presentes los siguientes aspectos:

- Una lectura crítica de la sociedad y de la educación predominante: La crítica de ideologías demanda una lectura del mundo que nos permita analizar la conflictividad inherente en la sociedad capitalista y la posición o rol que tenemos en dicha conflictividad. Si no se analiza la realidad sobre la que uno vive es muy probable que se caiga en una postura ideológica que conlleva a una práctica que adquiere un carácter de adoctrinamiento de los sectores populares. El interés de esta lectura es evidenciar las verdades que los sujetos y pueblos poseen, para desde allí, reconstruir y transformar dichas verdades y sus concreciones históricas (Carballo, 2008).

- La formación como emancipación: La formación política es entendida como acción liberadora. La educación busca la promoción del cambio social, en vez de la estabilidad social, es decir, es un instrumento para la transformación. Sin embargo, la EP no busca el cambio de unos por otros, es decir de explotados por explotadores, sino que busca superar dicha contradicción aspirando a una sociedad en la que no allá opresores ni oprimidos. El acento en la emancipación o liberación es que los seres humanos puedan desligarse de las dependencias que lo hacen ser "seres ara otros" antes que "seres para sí" (Torres,2011).

- Los sujetos como protagonistas de su emancipación: Lo popular en la EP no es un simple adjetivo, sino un sustantivo. De ese modo, lo popular en la EP tiene que ver con la intencionalidad y con el lugar social desde donde se realiza el proyecto. Con vistas a la creación de un poder popular que encarne los propios intereses de los sectores populares y no de quienes tienen intereses creados distintos a ellos. La emancipación no es una cosa que se deposita en los seres humanos. No es una palabra más, es praxis, que implica reflexión y acción transformadora (Mendoza y Nuñez, 2002).

- La subjetividad de los sujetos educativos como realización política y pedagógica: Esto se refiere a la concientización, a un proceso educativo - formativo que pretende lograr cambios en los pensamientos, en la lectura de la realidad y en la acción social. En este 
sentido, subjetividad es fundamentalmente recuperación de las tradiciones populares y las expresiones culturales populares (Lorenzo, 2008).

- Metodologías de trabajo apropiadas: implica un conjunto de técnicas participativas, activas y dialógicas. Así, se busca que la metodología de trabajo no sea a partir de los objetivos previamente trazados por otros sino que se busca un diálogo y un protagonismo de todos en la formación de una idea (Mejía y Awad, 2004).

- Relaciones existentes entre pedagogía y política: La relación entre lo pedagógico y lo político es íntima, las relaciones de saber son siempre relaciones de poder, por ello, la EP busca una acumulación de fuerzas para cambiar la situación de dominación e insiste sobre un trabajo, sobre una recomposición y un fortalecimiento de la sociedad civil. Se pone el acento sobre la organización de la base a partir del conocimiento de la realidad popular (Masi, 2008).

Por todo lo anterior, lo educativo es visto desde la EP, como una praxis pedagógica y política transformadora y orientada por sectores populares.

¿Qué es lo popular?.

Lo popular hace referencia a los sectores poblacionales subalternizados, son el conjunto plural de los grupos sociales explotados y excluidos de la administración de poder político moderno - colonial, como de la distribución de los excedentes económicos del sistema mundo. Lo popular, se define según relaciones de opresión tanto a nivel económico, como de género, de raza, de edad, entre otras. En esta perspectiva, la EP consiste en acompañar y relacionar diversas formas de saber y de organización para que los diferentes sujetos que participan en un mismo proyecto de transformación social se comuniquen entre sí y generen un poder contra - hegemónico (Masi, 2008).

En esta perspectiva, lo popular gira en torno a las siguientes coordenadas filosófico - politicas (García,1982,p.49):

- Apropiación desigual del capital cultural hegemónico: un problema de distribución y acceso a las ideas de mundo dominantes.

- Elaboración propia de sus condiciones de vida: la creación de formas de ser y estar en el mundo en clave de subalternidades. En este sentido, pueden existir formas de habitar el mundo que no son reconocidas por las hegemonias, que pueden existir en lo oculto o subterraneo, pero que no confrontan las hegemonías.

- Interacción conflictiva con los sectores hegemónicos. En este sentido lo subalterno deviene en contra hegemónico, cuando además de su alternativa particular de vida y acción, se propone un patrimonio común para enfrentar las ideas hegemónicas. Cuando se actúa local pensando global.

- Configuración de estrategias de alteración y alternativa al orden de cosas existente, con base en el diálogo de las múltiples subjetividades sublevadas.

\section{Conclusiones.}

Con las anteriores ideas y siguiendo a Dussel, se puede establecer diferencias entre el pueblo, el populismo y lo popular, tesis once de las veinte propuestas por Dussel (2006,Pp 87 -94). Así, el pueblo en la ideología estructuralista marxista deviene en una suerte de sujeto 
histórico idealizado que parte de la existencia previa a los sujetos de condiciones materiales de existencia, que son las encargadas de brindar legitimidad y coherencia a las ideas de clase llamadas a dominar. La conciencia de clase del proletariado sería la fuerza ideológica básica en la configuración política de la idea de pueblo. Para esta lectura el pueblo es una esencia previa a la acción humana. El populismo sería una manifestación deformada de la vocería del pueblo esencializado. El populismo es la construcción de una suerte de bloque histórico que no cuenta con las voces de los sujetos que establecen relaciones sociales y políticas de subalternidad. En este sentido, el populismo en nombre del pueblo comete una violencia simbólica sobre los que no poseen voz, dado que se da la potestad ideológica de hablar por ellos. Hablar en nombre de los que no tienen voz, es una acción antidialógica que cubierta por asistencialismo o autoritarismo niega la existencia de los otros. Por último, lo popular es el pueblo actuando, el pueblo siendo sujeto histórico con base en las anteriores coordenadas. A diferencia del pueblo esencializado, lo popular es el pueblo configurado en la lucha, en la resistencias y la contra hegemonía. A diferencia del populismo, lo popular no habla por, habla desde el pueblo en acción, no es la vocería de nadie, es la expresión legitima de la lucha social, de sus múltiples expresiones movimentistas (Mclaren, 2009).

Pensar la EP en clave pedagógico - política, es reivindicar ideas y acciones en torno a la educación y formación crítica en américa latina. Su pertinencia reside en la posibilidad de pensarnos localmente, de manera situada. Pensarnos, leernos y escribirnos es el reto que nos propone la EP.

La emancipación humana, la descolonización de nuestro ser es la propuesta formativa de la EP. Gracias a lo anterior es posible descolonizar el saber, nuestras maneras de nombrarnos, y descolonizar el poder, nuestras formas de relacionarnos y construirnos históricamente. 


\section{Referencias.}

Carballo Villagra, P. (2008). Una experiencia de educación popular según premisas de la pedagogía crítica. Obtenido de CLACSO:

http:/ / biblioteca.clacso.edu.ar/ar/libros/campus/freire/05Villarg.pdf.

Dussel, E. (2006). 20 tesis de politica. Mexico D.F.: Siglo XXI.

Fals Borda, O. (1970). Ciencia propia y colonialismo intelectual. Mexico D.F.: Nuestro Tiempo.

Freire, P. (1970). La educacion como practica de la libertad. Mexico D.F.: Siglo Veintiuno Editores .

Fuentes , N. (2008). Proyeccion de la propuesta de Paulo Freire. Obtenido de CLACSO: http:/ / biblioteca.clacso.edu.ar/ar/libros/campus/freire/30Fuent.pdf.

Garcia, N. (1982). Culturas populares en el capitalismo. Habana: Ediciones Casa de las Americas.

Lorenzo, Z. B. (2008). Educación popular, cultura e identidad desde la perspectiva de Paulo Freire. Obtenido de CLACSO:

http:/ / biblioteca.clacso.edu.ar/ar/libros/campus/freire/06Brito.pdf.

Masi, A. (2008). El concepto de praxis en Paulo Freire. Obtenido de CLACSO:

http://www.campusvirtuales.com.ar/campusvirtuales/comun/mensajes/206273 / 1/Concepto\%20de\%20Praxis\%20en\%20Freire.pdf.

Mclaren, P. (2009). El Che Guevara, Paulo Freire y la pedagogia de la revolución. Buenos Aires: Siglo Veintiuno Editores.

Mejia, M., \& Awad, M. (2004). La educacion popular hoy.En tiempos de Globalizacion. Bogota: Ediciones Aurora.

Mendoza, C., \& Nuñez, C. (2002). Educación popular y los formadores políticos. Obtenido de Incep:

http://www.bantaba.ehu.es/formarse/ficheros/view/metodologia_de_la_eduacion _popular.pdf?revision_id=80144\&package_id=80083.

Torres, A. (2011). La educacion popular.Trayectoria y actualidad. Caracas: Imprenta Universitari UBV. 
${ }^{2}$ Este texto hace parte del proyecto de investigación titulado: De Tijuana a la Patagonia: perspectivas críticas en educación en América Latina. Financiado por la USB - Medellín. 